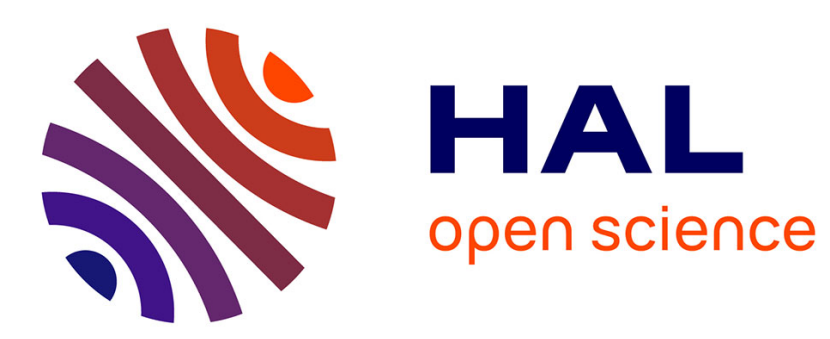

\title{
New fluid approaches for studying the performance of elastic traffic under class based weighed fair queuing system
}

\author{
Mohamed El Hedi Boussada, Jean Marie Garcia, Mounir Frikha
}

\section{To cite this version:}

Mohamed El Hedi Boussada, Jean Marie Garcia, Mounir Frikha. New fluid approaches for studying the performance of elastic traffic under class based weighed fair queuing system. International Journal of Network Management, 2018, 28 (6), pp.e2037. 10.1002/nem.2037 . hal-01888319

\section{HAL Id: hal-01888319 \\ https://hal.science/hal-01888319}

Submitted on 8 Nov 2018

HAL is a multi-disciplinary open access archive for the deposit and dissemination of scientific research documents, whether they are published or not. The documents may come from teaching and research institutions in France or abroad, or from public or private research centers.
L'archive ouverte pluridisciplinaire HAL, est destinée au dépôt et à la diffusion de documents scientifiques de niveau recherche, publiés ou non, émanant des établissements d'enseignement et de recherche français ou étrangers, des laboratoires publics ou privés. 
DOI: $\mathrm{xxx} / \mathrm{xxxx}$

\title{
ARTICLE TYPE
}

\section{New Fluid Approaches For Studying the Performance of Elastic Traffic under Class Based Weighed Fair Queuing System}

\author{
Mohamed El Hedi Boussada*1 | Jean Marie Garcia² | Mounir Frikha ${ }^{3}$
}

\author{
${ }^{1}$ MEDIATRON Lab, SUP'COM, University \\ of Carthage, Ariana, Tunisia \\ ${ }^{2}$ Services and Architectures for Advanced \\ Networks, LAAS-CNRS, Toulouse, France \\ ${ }^{3}$ MEDIATRON Lab, SUP'COM, University \\ of Carthage, Ariana, Tunisia \\ Correspondence \\ *Mohamed El Hedi Boussada, \\ MEDIATRON Lab, SUP'COM, University \\ of Carthage,Ariana-Tunisia. Email: \\ med.elhadi.boussada@supcom.tn
}

\begin{abstract}
Summary
Although TCP is typically designed to carry data (transfer of documents such as files or web pages), it is also suitable, today, for transporting most commercial video streaming traffic such as YouTube or Netflix traffic. The Class-Based Weighted Fair Queuing (CBWFQ) is still an important router discipline that allows different classes of elastic flows (generally TCP flows) to be transported together in a truly converged network. Such system has been extensively studied in packet level by evaluating several criteria of effectiveness such as the mean queue length and the average queue waiting time, without proposing a general model that captures the flow-level dynamics and the real coupling aspect between different queues. Moreover, most studies limited their works to a simple two-queue system where performance evaluation is very much easier. Even the few works focusing on providing extended results did not give accurate results for very loaded systems. Proposed packet-level models seem to be not convenient to predict the performance of large-scale operator networks with millions of users, millions of flows and unexpected user behaviours. This paper aims to overcome these limitations by presenting new analytical explicit mathematical expressions evaluating the flow-level performance metrics of elastic traffic under a general CBWFQ system. The core of our analysis is based on some approximations proven for balanced fairness allocation, which provides a reasonable framework for estimating bandwidth sharing among elastic traffic for best-effort allocations. Detailed packet level simulations are used to verify the accuracy and the effectiveness of the two proposed approaches.
\end{abstract}

\section{KEYWORDS:}

Class-Based Weighted Fair Queuing, elastic traffic, flow level, balanced fairness, Quality of Service

\section{1 | INTRODUCTION}

Upgrades in Internet connection speeds, with the emergence of 4G LTE cellular and fiber optic communications ${ }^{112}$, has led to a multiplication of services offered by modern telecommunication networks and to unprecedented growth in the number of users and traffic volumes that they generate ${ }^{2 / 3 / 4}$. Internet traffic is forecasted to increase nearly threefold over the next five years. Major portion of this traffic will be mainly dedicated to various forms of video. Globally, IP video traffic will be 82 percent of all consumer Internet traffic by $2021^{5}$. Although Internet tends progressively for interactivity, it is anticipated that non-online video applications will still contribute by the most significant amount of video traffic $\underline{6}$. These applications are usually based on 
TCP (Transport Control Protocol). It is reported that most commercial streaming traffic (YouTube, Facebook, Netflix, etc.) is carried over $\mathrm{TCP} 7819$.

Since these applications rely on TCP for packet transmission, the generated traffic is elastic in nature. This is because TCP's congestion control adapts to the available capacity in the network and results in an elastic packet transmission rate ${ }^{10}$. The adaptive nature of elastic traffic makes it more tolerant to delay and jitter than real-time traffic ${ }^{11}$. However, given that video applications are generally exigent in terms of bandwidth, TCP-based video traffic should be not transported in the same manner as classical TCP traffic (file transfer, web pages, mailing). Operators and service providers generally offer all services at different service levels according to the Quality of Service (QoS) level required by both the service and the client ${ }^{2}$. For example, the Service Level Agreement (SLA) created between the operator and the client is presented in three classes: bronze, silver, and gold levels with increasingly better response times. A service level is used to define the expected performance behaviour of a service, where the performance metrics are, for example, average response time, supported throughput, service availability, etc $\frac{12}{\text { ? }}$.

The Diffserv architecture presents an architecture where packets are marked and divided into service classes as they enter the network $\frac{13}{13}$. Network nodes are then able to provide different services using some scheduling algorithms. The Class-Based Weighted Fair Queuing (CBWFQ) is a scheduling discipline usually applied to QoS enabled routers ${ }^{14}$. CBWFQ is based on Weighted Fair Queuing (WFQ) scheduling. However, in contrast with WFQ, which specifies the capacity fraction to be given for each single flow, the CBWFQ policy allows the creation of user-defined flow classes, each of which is assigned to one specific queue with a specific weight that determines the guaranteed bandwidth for this class of flows. Each queue can use more bandwidth if it is available: When one class does not use all its guaranteed bandwidth, the other classes will share this residual capacity, which creates a coupling aspect between queues in order to optimize the resource sharing and ensure a more efficient traffic transport. This scheduling discipline is very important to understand how heterogeneous elastic traffic converges and competes for the use of the same-shared transmission resources. CBWFQ may be able to meet the QoS measures needed by the different elastic traffic types 15 .

Although the issue of resource allocation in CBWFQ system can't be addressed without taking into account the random and the dynamic nature of ongoing flows ${ }^{16}$, studying the flow level performance of elastic traffic under such system (or an equivalent system) has not received much attention in literature. CBWFQ system has been extensively studied at packet level by evaluating several criteria of effectiveness such as the mean queue length and the average queue waiting time $14|15| 17|18| 1920 \mid 21$, without proposing a general model that captures the flow-level dynamics (arrivals and departures of flows) and the real coupling aspect between different queues.

The real behavior of a general CBWFQ system is hard to be analyzed under Markov models. In the presence of variable service rate (due to the coupling aspect existing between the different queues), it is very difficult to have a regular representation of the generating matrix of the Markov chain modelling the arrival and the departure of jobs/packets/flows in such system. One raison is that this generating matrix must combine all possible queue states, which is not always easily feasible. To keep a manage-

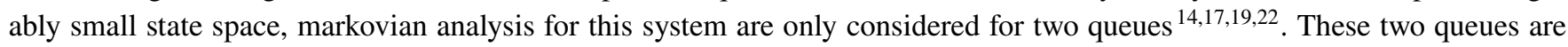
approximated by independent working servers with variable instantaneous service rates such that the service rate of one queue depends on the state of the other queue.

For a larger number of queues, some approximations were proposed as an alternative of Markov chain analysis. $\operatorname{In}^{21}$, the authors generalise the decoupling approach to a greater number of queues. However, their approach does not give an accurate results when the queue is strongly coupled to the other queues (a queue with low weight). Another numerical-based approach was proposed in ${ }^{[15}$, where the authors consider that coming packets will be assigned to the queues under a fixed probability. This probability is numerically deduced from system parameters and it depends on queue's weight. For very loaded system, this approach is far from being accurate.

Proposed packet-level models seem then to be not convenient to predict the performance of large-scale operator networks with millions of users, millions of flows and unexpected user behaviors. This paper aims to overcome these limitations by presenting new analytical results to evaluate the performance of elastic traffic under CBWFQ system. The idea behind our analysis is to exploit some approximations proven for balanced fairness allocation, which is considered as an efficient tool to approximate bandwidth sharing among elastic traffic for best-effort allocations. Using these approximations, we give two different fluid approaches for CBWFQ system: the first is a classical decomposition of the system into different links (virtual links) characterized each by an average capacity to be calculated while the second is based on some numerical results, issued from event 
simulations, that capture the evolution of the average number of flows per queue in function of the weight assigned to it. The originality of our contribution consists on providing explicit analytical expressions to predict the flow-level performance metrics (average throughput per flow, average number of connections for each class of flows, average total number of flows passing through one queue) of elastic traffic under CBWFQ system taking into account the strong coupling between the queues. To our knowledge, exact or approximated results do not exist for the model described in this paper.

The rest of this paper is organised as follows: an overview of related work is shown in the next section. In the third section, we describe our model of the system to be studied. In the fourth section, we give useful approximations proven for balanced fairness. We discuss then the bandwidth sharing between elastic traffic for the two cases where all flow classes have identical or different rate-limits. These approximations will be exploited in the fifth section to evaluate the performance of a general CBWFQ system. The accuracy of the proposed analytic results is discussed in the last section basing on some detailed packet level simulations with Network Simulator (NS 2).

\section{2 | RELATED WORK}

In this section, we first present the main difference between packet-level modelization and flow-level modelization focusing on some flow-level models evaluating the performance of elastic Internet traffic. Then, we discuss previous works studying the CBWFQ system or other variants of such system such as WFQ system. The difference between WFQ and CBWFQ is that CBWFQ differentiates traffic by class (e.g., voice, data, or video) while WFQ differentiates traffic by flow (e.g., source and destination). WFQ offers fair queuing that divides the available bandwidth across active flows based on weights. Each flow is associated to an independent queue with a specific weight, which determines the minimum percentage of bandwidth to be exploited by this flow. CBWFQ is an advanced form of WFQ that supports user defined traffic classes. A queue is allocated for each class of flows, and the traffic belonging to that class is directed to the queue assigned to $\mathrm{it}^{23}$. In CBWFQ policy, the weight of the queue indicates the guaranteed capacity for each traffic class.

\subsection{Flow level modelization of elastic traffic}

The performance evaluation of elastic traffic is a topic treated in the literature basically under two different levels: packet level and flow level ${ }^{24}$.

The packet level defines the way in which packets are generated and transported during the communication 25 . The packet level models incorporate many details about the system (Round Trip Times, buffer size, etc.) ${ }^{26}$. However, they generally consider a fixed number of 'long-lived' flows. These models may be useful to estimate queue sizes at buffers and packet loss, but they are still be considered as static models since they do not capture the flow-level dynamics, such as flow duration or the variation of the active number of persistent flows. Flow-level models are idealized models that include random flow-level dynamics (arrivals and departures of flows) and use highly simplified models of bandwidth sharing 26 . The complex underlying packet-level mechanisms (congestion control algorithms, packet scheduling, buffer management,etc.), at short time scales, are then simply represented by a long-term bandwidth sharing policy between ongoing flows 16127. The flow-level models of data networks can be considered as the analogues for the Erlang model of telephone networks and its extensions to multi-rate circuit-switched networks, which have proved its effectiveness for both dimensioning and traffic engineering 28 .

In general, a flow is defined as a series of packets between a source and a destination having the same transport protocol number and port number ${ }^{29}$. We refer to class of flows as all flows of the same service between a source and a destination, having a common rate limitation and the same resource requirements ${ }^{30}$.

The dynamic flow level modelling of Internet traffic was practically introduced with Massoulié and Roberts with a model of

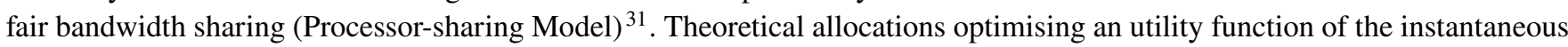
flow rates have also been proposed to estimate the TCP bandwidth sharing. Examples of such allocations are the classical max-min fairness and the Kelly's proportional fairness ${ }^{32}$. In general, the analysis of a network operating under these allocations schemes is quite difficult. One reason is that they do not lead to an explicit expression for the steady state distribution, which determines the typical number of competing flows of each class ${ }^{27}$. For this reason, balanced fairness was proposed by Bonald and Proutière to approximate the performance of TCP traffic under these equitable allocations $\frac{16 / 33134}{}$. A key property of 
balanced fairness is its insensitivity: the steady state distribution is independent of all traffic characteristics beyond the traffic intensity $1627 / 33$. The only required assumption is that flows arrive as a Poisson process. The average number of active flows can be then evaluated for each class. According to Little's law, this metric is essential to deduce the performance metrics of TCP traffic at flow level such as the average throughput and average transmission delay per flow within each class.

Nevertheless, the balanced fairness allocation remains complex to be used in a practical context as it requires the calculation of the probability of all possible states of the system, and thus it faces the combinatorial explosion of the state space for large networks 32 35136. In ${ }^{32}$, Bonald et al. propose a recursive algorithm to evaluate performance metrics in a tree networks, in which it is possible to identify congested network links for each system status. Although this algorithm makes it possible to calculate accurate performance metrics, it is applicable only for this particular case of network topologies. For more complex networks, identification of saturated links is not always feasible ${ }^{35}$. Another approach has been proposed in ${ }^{37}$ by Bonald et al. to resolve this problem. Under the assumption that flows do not have a rate limit (A flow may use all the link bandwidth if there is no flows in the same link), the authors propose explicit approximations of key performance metrics in any network topology. In practice, flows generally have a peak rate that is typically a function of the user access line.

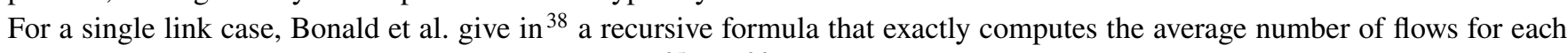
class. A reformulation of this formula was given in ${ }^{35}$ and ${ }^{36}$ where authors aim to give an explicit exact expression for the average number of flows per class. In order to reduce the complexity (and then the calculation's time), the authors provided an approximation to their original expression. This approximation was generalised after for all network topologies $\frac{35[36 / 39}{}$. Flow level performance metrics for elastic traffic become then easier to be predicted and evaluated for large-scale networks. We will introduce these approximations later, because they are very useful to study our present system.

\subsection{An overview of related work studying the CBWFQ system}

Most works studying the CBWFQ system (or WFQ system) focus on modelling this system at packet level by evaluating several criteria of effectiveness for this level such as the average queue waiting time and mean queue length 14 |15/17/18/19/20|21.

The real behavior of a general CBWFQ system is hard to be exactly modelled, especially because of the coupling aspect existing between the different queues. In the presence of variable service rate, it is very difficult to have a regular representation of the generating matrix of the Markov chain modelling the arrival and the departure of jobs/packets/flows in such system as it combines all possible queue states 21 . To keep a manageably small state space, Markovian analysis for this system, or an

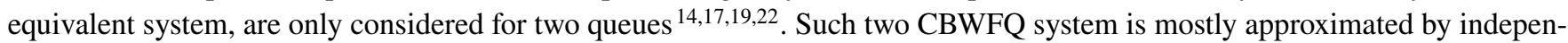
dent working servers with variable instantaneous service rates ${ }^{22 / 40 / 4142}$. The instantaneous available service rate is generally considered as the minimum service rate for a queue, calculated by multiplying the weight of the queue by the server capacity, plus the remaining service rate from the other queue.

For large number of queues, some approximate techniques are proposed as an alternative to Markov chain analysis. $\mathrm{In}^{21}$, authors aim to generalise the decoupling approach for more than two queues. However, for reason of simplicity, they only consider the first-level calculation of the remaining service rate. It means that the remaining service rate from a queue, given that it is already using the remaining service rate form other queues, is not treated. Authors show that this viewpoint is unable to capture the strong coupling between queues and it can't always provide accurate results. In $\frac{15}{15}$, another approach was proposed. Authors started their study by modelling a two CBWFQ system as a non-preemptive priority system, where packets of each class are randomly assigned to one of priority queues according to a fixed probability. This probability was fixed based on experimental simulations and it is in function of the class'weight. Then, they extended their results to more complex queuing scenarios. The proposed results work very well for small overall utilisation of the system. However, it seems to be not very accurate for medium and high values of total load, where the error rate exceeds $10 \%$ and reaches $40 \%$ for high traffic demand. In general, the CBWFQ policy has practically no effect for small load ${ }^{43}$. Therefore, an efficient approximation for a CBWFQ system should especially give reasonable results for medium and high values of total load.

To conclude, the main limitations of the different analysis proposed for evaluating the performance of a CBWFQ system (or an equivalent system) can be summarised in these two points:

1. The proposed models didn't capture the flow level dynamics (arrival and departure of flows), which represent very well the great dynamicity of the traffic as it makes it possible to represent the connections and disconnections of users on an application 
(or service).

2. The proposed models couldn't capture the coupling aspect between queues in a general CBWFQ system.

In this paper, we aim to provide new fluid approaches to overcome these two limitations. The originality of our contribution consists on:

1. Providing a large-scale modelization of the CBWFQ system, taking into account the strong coupling between the queues.

2. Evaluating the performance of elastic traffic under this system basing on some analytical results already proven for a simple best-effort system.

\section{3 | MODEL}

We consider a large number of ADSL, UMTS or LTE subscribers distributed over a single geographic area and connected to the backbone network through an access line of capacity $C$ (Mbits/Second). This access line is assumed to be the bottleneck link for all the traffic flows generated by users. This link is a full-duplex link, which means that the transmission capacity is $C$ (M bits/Second) in both directions.

Users generate a random number of elastic flow-classes. Let $E$ be the set of these elastic flow classes. Class- $i$ flows, $i \in E$, arrive as an independent Poisson process with rate $\lambda_{i}$ (Flows/Second) and have independent exponentially distributed volumes with mean $\sigma_{i}($ M bits $/$ Flow $)$. We refer to the product $\rho_{i}=\lambda_{i} \sigma_{i}$ (M bits $/$ Second $)$ as the traffic intensity of class $i$. Let $\theta=\sum_{i \in E} \rho_{i}$ be the total traffic intensity generated by all elastic flows. We assume that $\theta<C$ to ensure the stability of our system.

Each flow of a class $i$ has a maximum bit rate $d_{i} \leq C$ in $(M$ bits $/$ Second $)$. This is the actual rate of each flow in the absence of congestion. Congestion forces TCP to reduce flow rates and thus to increase their durations. Let $x_{i}$ the number of class- $i$ flows. We refer to the vector $x=\left(x_{i}\right)_{(i \in E)}$ as the network state, the vector $\rho=\left(\rho_{i}\right)_{(i \in E)}$ as the vector of traffic intensities and the vector $d=\left(d_{i}\right)_{(i \in E)}$ as the vector of rate limits.

In a similar way to the configuration of Internet routers, we assume that, for both directions, packets are assigned to a number $M$ of CBWFQ queues before being sent into the link. Let $w_{m}, m=1 . . M$, the weight of the CBWFQ queue number $m$. We assume that:

$$
\sum_{m=1}^{M} w_{m}=1
$$

We denote by $E_{m}$ the set of elastic flow classes passing through the queue number $m$ and by $\theta_{m}=\sum_{i \in E_{m}} \rho_{i}$ the load offered to this queue. Let $x^{(m)}=\left(x_{i}\right)_{\left(i \in E_{m}\right)}$ be the state of the queue number $m$ and $d^{(m)}=\left(d_{i}\right)_{\left(i \in E_{m}\right)}$ be the vector of limit rates for this queue. Since the above resource sharing is applied for both upload and download directions, our study will be limited to one CBWFQ system. The evolution of this system state defines a multidimensional Markov process with transition rates $\lambda_{i}$ from state $x$ to state $x+e_{i}$ and $d_{i}(x) / \sigma_{i}$ from state $x$ to state $x-e_{i}$ (provided $x_{i}>0$ ), where $d_{i}(x)$ is the bit rate of class- $i$ flows in a state $x$ $\left(d_{i}(x) \leq d_{i}\right)$ and $e_{i}$ represents a unit vector with 1 in position $i$, and 0 elsewhere. If we denote by $\phi_{m}(x)$ the bandwidth given to the queue $m$ by the CBWFQ policy in a state $x$, and supposing, for example, that each queue is exploited by identical limit rate flows, $d_{i}(x)$ will be given by $2 \forall i \in E_{m}$.

$$
d_{i}(x)=\min \left(d_{i}, \frac{\phi_{m}(x)}{\sum_{i \in E_{m}} x_{i}}\right)
$$

It is very difficult to give an explicit expression to $\phi_{m}(x)$ for each state $x$ given the coupling existing between the queues. Since the weight for each queue indicates the minimum capacity that the queue will receive, we have :

$$
\phi_{m}(x) \geq w_{m} C
$$

In particular, if a state $x$ is characterised by $\sum_{i \in E_{k}} x_{k} d_{k} \geq w_{k} C$ for all queue $1 \leq k \leq M$, the expression 3 becomes:

$$
\phi_{m}(x)=w_{m} C
$$

For elastic traffic, we assume that users perceive performance essentially through the average throughput per flow given by 5 for each $i \in E^{[16] 27] 33] 35}$.

$$
\gamma_{i}=\frac{\rho_{i}}{E\left[x_{i}\right]}
$$

Where $E\left[x_{i}\right]$ is the average number of active flows for the class $i$. 
In the following, we use these notations:

$$
\begin{gathered}
|x|=\sum_{i \in E} x_{i} \\
\rho^{x}=\prod_{i \in E} \rho_{i}^{x_{i}} \\
x d=\sum_{i \in E} x_{i} d_{i}
\end{gathered}
$$

We also denote by $\pi(B)$ the stationary probability of a set $B$ and by $\pi(x)$ the stationary probability of the vector $x$.

\section{4 | APPROXIMATE RESULTS FOR BALANCED FAIRNESS ALLOCATION}

In this section, we assume that there is no CBWFQ policy in the entry of the link (which is equivalent to letting $M=1$ in our model). The objective of this section is to introduce some useful approximations for balanced fairness allocation in order to easily estimate the performance of elastic traffic in best-effort system. These approximations will be exploited in the next section to evaluate the performance of our studied system.

\section{1 | Identical rate limits}

We suppose here that all classes have the same maximum bit rate $r$. Let $N=\lfloor C / r\rfloor$ be the maximum number of flows that can have their maximum bit rate. $\mathrm{In}{ }^{[35}$, Ben Cheikh proved that, under balanced fairness allocation, the average number of flows for each class $i$ is exactly given by:

$$
E\left[x_{i}\right]=\frac{\rho_{i}}{r}+\pi(B) \frac{\rho_{i}}{C-\theta}
$$

Where $B=\{x,|x| \geq N\}$ is the set of congestion stats:

$$
\pi(B)=\frac{\left(\frac{\theta}{r}\right)^{N}}{N !} \frac{C}{C-\theta} \pi(0)
$$

$\pi(0)$ is the probability that the link is empty, and it is given by:

$$
\pi(0)=\left(\sum_{k=0}^{N-1} \frac{\left(\frac{\theta}{r}\right)^{k}}{k !}+\frac{\left(\frac{\theta}{r}\right)^{N}}{N !} \frac{C}{C-\theta}\right)^{-1}
$$

The average total number of flows can be then deduced from 6 by:

$$
E[x]=\frac{\theta}{r}+\pi(B) \frac{\theta}{C-\theta}
$$

And then, we have the following relation:

$$
E\left[x_{i}\right]=\frac{\rho_{i}}{\theta} E[x]
$$

\subsection{General rate limits}

For different transmission rates, an approximation was given in ${ }^{\sqrt[35]{, 36}}$ and ${ }^{\sqrt[39]{39}}$ to simplify the exact expression (presented essentially in $\frac{38}{}$ as a recursive formula) of the average number of flows for each class of flows $i \in E$ under balanced fairness allocation:

$$
E\left[x_{i}\right] \approx \frac{\rho_{i}}{d_{i}}+\pi\left(B_{i}\right) \frac{\rho_{i}}{C-\theta}
$$

Where $B_{i}$ is the set of congestion stats for the class $i: B_{i}=\left\{x, x d>=C-d_{i}\right\}$. The probability $\pi\left(B_{i}\right)$ is written as follows:

$$
\pi\left(B_{i}\right)=\frac{1}{C-\theta} \sum_{j \in E} \rho_{j} \pi\left(W_{j}\right)+\pi\left(W_{i}\right)
$$


$W_{j}$ is defined as $W_{j}=\left\{x, C-d_{i} \leq x d<C\right\}$ and its probability is given by:

$$
\pi\left(W_{j}\right)=\pi(0) \sum_{x \in W_{j}} P(x d, C)
$$

Where $P(x d, C)$ is given by the Kaufman-Roberts formula. This formula can be reformulated as follows:

$$
P(n, C)=\sum_{k \in E} \frac{\rho_{k}}{n} P\left(n-d_{k}, C\right) \quad \forall 1 \leq n \leq C
$$

with $P(0, C)=1$ and $P(n, C)=0$ for all $n<0$.

The probability that the link is empty is then given by:

$$
\pi(0)=\left(\sum_{0 \leq x d<C} P(x d, C)+\frac{1}{C-\theta} \sum_{j \in E} \rho_{j} \sum_{x \in W_{j}} P(x d, C)\right)^{-1}
$$

In our following analysis, we will use this approximation rather than the exact recursive formula of Bonald for two main reasons. One is that this approximation is less complex and provide a very accurate results (see Figure 1 . The other is that this approximation was generalised to the case of any network topology, which make our results easily scalable in the future.

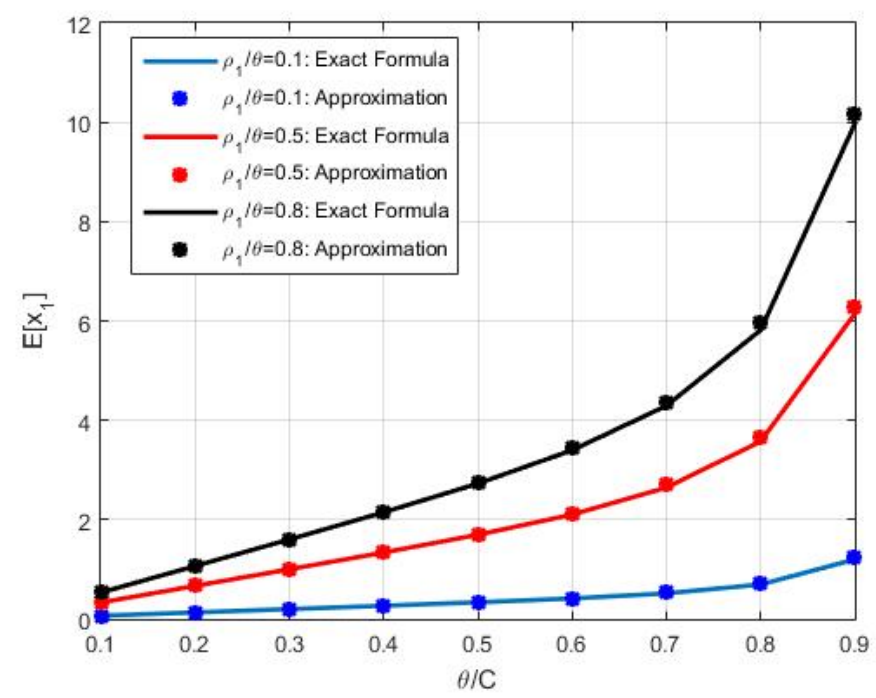

FIGURE 1 Comparison in terms of $E\left[x_{1}\right]$ between the exact recursive formula presented in ${ }^{38}$ and the approximation 11 for different values of $\rho_{1} / \theta$ : Example of a link of capacity $C=20$ shared by three classes of flows such as: $d_{1}=1, d_{2}=2, d_{3}=3$ and $\rho_{2} / \theta=\rho_{3} / \theta$.

\section{5 | STUDYING THE CBWFQ SYSTEM}

The capacity $w_{m} C$ is mainly dedicated to flows passing through the queue $m$. However, if a part of this capacity remains available, it will be shared between the other virtual links, which need more resources to transmit its traffic in a better condition. Therefore, $w_{m} C$ can be viewed as the minimum capacity allocated to the virtual link number $m$. Bonald et al. implicitly expressed this idea in ${ }^{43}$ saying that the QoS offered to flows is always better than that obtained if the link were divided into $M$ virtual links of capacity $w_{m} C, 1 \leq m \leq M$.

In this section, we will exploit the results presented in the previous section to capture this coupling between the queues and provide analytical explicit results for flow level performance metrics of elastic traffic. We analyse the CBWFQ system under two different approaches: the first is a classical decomposition of the system into different independent virtual links, each with an 
average capacity to be calculated. The second one treats the whole system as an one entity (without decoupling it) and it relies on some numerical observations of the evolution of the average total number of flows for a queue in function of its weight.

\section{1 | First approach: Decoupling of the system into independent virtual links}

We suppose that $\theta_{m}<w_{m} C, \forall m$. Our first approach is based on decomposing the system into different independent virtual links characterized each by an average capacity value. This average capacity reflects the mean service rate that a queue will receive during all states of the system. Then, we independently evaluate the performance of each queue using 6 and 11

It is evident that, in some states of $x$, one or more queues (or virtual links) may not use the full capacity initially dedicated to it. This remaining capacity can be used by other queues, according to their weights, to increase the total queue service rate for better QoS. Calculating the mean capacity of each virtual link is then given by the enumeration of all possible states. After all calculations have been made, we propose the following expression for the average capacity of each virtual link $m$ :

$$
\overline{C_{m}}=w_{m} C+\alpha_{m}
$$

It is clear that $\alpha_{m}$ is the average capacity received by the queue $m$ from the other queues. It is given by:

$$
\alpha_{m}=\alpha_{m}^{(1)}+\alpha_{m}^{(2)}
$$

Where the $\alpha_{m}^{(1)}$ gives the average capacity received from the other queues when all other queues do not use its minimum capacity and $\alpha_{m}^{(2)}$ gives the average capacity received when there is at least one other queue that will share the total remaining capacity with the queue $m$. We prove that (See APPENDIX A):

$$
\alpha_{m}^{(1)}=\left(1-w_{m}\right) C \prod_{\substack{q=1 \\ q \neq m}}^{M}\left[1-\pi\left(B^{(q)}\right)\right]-\sum_{\substack{k=1 \\ k \neq m}}^{M} \sum_{i \in E_{k}} \rho_{i}\left[1-\pi\left(B_{i}^{(k)}\right)\right] \prod_{\substack{q=1 \\ q \neq k \\ q \neq m}}^{M}\left[1-\pi\left(B^{(q)}\right)\right]
$$

and (See APPENDIX B):

$$
\alpha_{m}^{(2)}=\sum_{t=1}^{M-2}\left[\sum_{\substack{i_{1}=1 \\ i_{1} \neq m}}^{M-(t-1)} \sum_{\substack{i_{2}=i_{1}+1 \\ i_{2} \neq m}}^{M-(t-2)} \sum_{\substack{i_{t}=i_{t-1}+1 \\ i_{t} \neq m}}^{M} C_{\left(t, i_{q, q 1} . t\right)}\right]
$$

With:

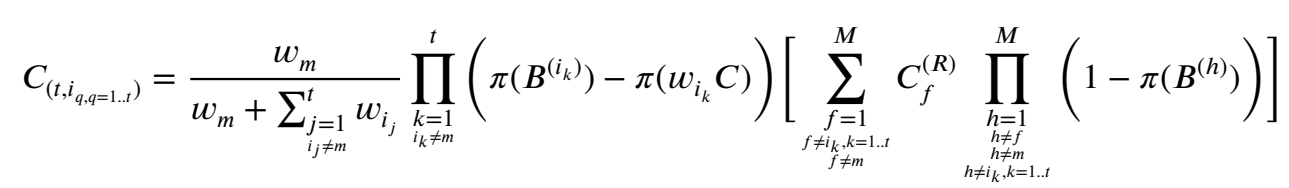

$\pi\left(w_{i_{k}} C\right)$ gives the probability that the traffic passing through the queue $i_{k}$ requires a bandwidth exactly equal to $w_{i_{k}} C$ :

$$
\pi\left(w_{i_{k}} C\right)=\pi(0)^{i_{k}} P\left(w_{i_{k}} C, w_{i_{k}} C\right)
$$

For each queue $q, \pi(0)^{(q)}$ is deduced from 15 as follows:

$$
\pi(0)^{(q)}=\left(\sum_{0 \leq x^{(q)} d^{(q)}<w_{q} C} P\left(x^{(q)} d^{(q)}, w_{q} C\right)+\frac{1}{w_{q} C-\theta_{q}} \sum_{j \in E_{q}} \rho_{j} \sum_{x^{(q)} \in W_{j}^{(q)}} P\left(x^{(q)} d^{(q)}, w_{q} C\right)\right)^{-1}
$$

The set $W_{j}^{(q)}$ is defined as $W_{j}^{(q)}=\left\{x^{(q)}, w_{q} C-d_{j} \leq x^{(q)} d^{(q)}<w_{q} C\right\}$. The probability of this set is given by 13 by replacing $C$ with $w_{q} C, x d$ with $x^{(q)} d^{(q)}, \theta$ with $\theta_{q}$ and eventually $W_{j}$ with $W_{j}^{(q)}$.

For each queue $q, \boldsymbol{B}^{(q)}$ is defined as: $\boldsymbol{B}^{(q)}=\left\{x^{(q)}, x^{(q)} d^{(q)} \geq w_{q} C\right\}$. The probability $\pi\left(\boldsymbol{B}^{(q)}\right)$ is deduced from 12 as follows:

$$
\pi\left(B^{(q)}\right)=\frac{1}{w_{q} C-\theta_{q}} \sum_{j \in E_{q}} \rho_{j} \pi\left(W_{j}^{(q)}\right)
$$


$C_{f}^{(R)}$ is the average remaining capacity in the virtual link number $f$. Given that the capacity $w_{f} C$ of each virtual link $f$ is shared according to balanced fairness among the different elastic flow classes in $E_{f}$, we prove that, $C_{f}^{(R)}$ is given by (See APPENDIX C):

$$
C_{f}^{(R)}=w_{f} C\left[1-\pi\left(B^{(f)}\right)\right]-\sum_{i \in E_{f}} \rho_{i}\left[1-\pi\left(B_{i}^{(f)}\right)\right]
$$

For each queue $q$, and for each $i \in E_{q}$, the probability of the set $B_{i}^{(q)}=\left\{x^{(q)}, x^{(q)} d^{(q)} \geq w_{q} C-d_{i}\right\}$ is given from 12 as follows:

$$
\pi\left(B_{i}^{(q)}\right)=\frac{1}{w_{q} C-\theta_{q}} \sum_{j \in E_{q}} \rho_{j} \pi\left(W_{j}^{(q)}\right)+\pi\left(W_{i}^{(q)}\right)
$$

If all flows passing through a queue $f$ have the same maximum bit rate $r^{(f)}$, the expression of $C_{f}^{(R)}$ can be simply written as follows (See APPENDIX D):

$$
C_{f}^{(R)}=\left(w_{f} C-\theta_{f}\right)\left[1-\pi\left(B^{(f)}\right)\right]+w_{f} C \pi\left(\left|x_{f}\right|=N^{(f)}\right)
$$

Where $\pi\left(B^{(f)}\right)$ is deduced from 7 as follows:

$$
\pi\left(B^{(f)}\right)=\pi\left(\left|x_{f}\right| \geq N^{(f)}\right)=\frac{\left(\frac{\theta_{f}}{r^{(f)}}\right)^{N^{(f)}}}{N^{(f)} !} \frac{w_{f} C}{w_{f} C-\theta_{f}} \pi(0)^{(f)}
$$

With:

$$
N^{(f)}=\left\lfloor\frac{w_{f} C}{r^{(f)}}\right\rfloor
$$

and:

$$
\pi(0)^{(f)}=\left(\sum_{k=0}^{N^{(f)}-1} \frac{\left(\frac{\theta_{f}}{r^{(f)}}\right)^{k}}{k !}+\frac{\left(\frac{\theta_{f}}{r^{(f)}}\right)^{N^{(f)}}}{N^{(f)} !} \frac{w_{f} C}{w_{f} C-\theta_{f}}\right)^{-1}
$$

$\pi\left(\left|x_{f}\right|=N^{(f)}\right)$ is given by:

$$
\pi\left(\left|x_{f}\right|=N^{(f)}\right)=\frac{\left(\frac{\theta_{f}}{r^{(f)}}\right)^{N^{(f)}}}{N^{(f)} !} \pi(0)^{(f)}
$$

The system is then decoupled into $M$ independent virtual links, each with an average capacity $\overline{C_{m}}, m=1 . . M$. The mean number of each class $i \in E_{m}$ can be given according to 11 as follows:

$$
E\left[x_{i}\right] \approx \frac{\rho_{i}}{d_{i}}+\pi\left(B_{i}^{(m)^{*}}\right) \frac{\rho_{i}}{\overline{C_{m}}-\theta_{m}}
$$

Where $B_{i}^{(m)^{*}}=\left\{x^{(m)}, x^{(m)} d^{(m)} \geq \overline{C_{m}}-d_{i}\right\}$. The probability of this set is given from 12 by replacing $\theta$ with $\theta_{m}, C$ with $\overline{C_{m}}$, $x d$ with $x^{(m)} d^{(m)}$ and eventually $E$ with $E_{m}$. In the case when all flows passing through a queue $m$ have an identical maximum bit rate $r^{(m)}$, the expression of $\pi\left(B^{(m)^{*}}\right)$ is given from 7 by replacing $C$ with $\overline{C_{m}}, r$ with $r^{(m)}, \theta$ with $\theta_{m}$ and eventually $N$ with $N^{(m)^{*}}=\left\lfloor\overline{C_{m}} / r^{(m)}\right\rfloor$.

As already mentioned at the beginning of this section, this approach is based on assuming that all virtual links must initially be in a state of stability, which means that the intensity of traffic carried by each virtual link must be strictly inferior to its minimum capacity. Indeed, this assumption is fundamental in order to use the formulas presented in section 4 . This is the main drawback of this approximation. In a real context, it is sufficient to check that the system has an overall stability, where the total incoming traffic intensity must not exceed the link's capacity.

As a simplifying assumption, we can admit here that if the traffic intensity of a given virtual link $m$ exceeds the minimum capacity dedicated to it, the capacity $w_{m} C$ is supposed to be always occupied and flows crossing it have a very low probability to have their maximum rates. Similarly, if the average total load offered to a virtual link is still superior to its average capacity, we admit that the average end-to-end throughput of all flows passing through this virtual link rapidly tends to zero. This hypothesis is justified by the fact that when a given link confronts an instability, the number of flows passing through it indefinitely increases and thus the quality of service of these flows is progressively degraded. 


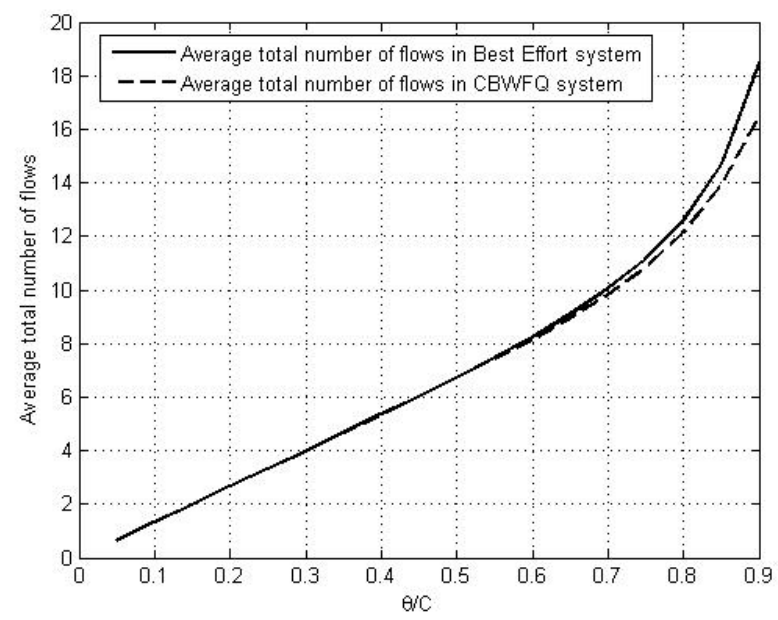

FIGURE 2 Comparison between the best-effort system and CBWFQ system in terms of the average total number of flows.

\section{2 | Second Approach: Treating the CBWFQ system as an one entity basing on numerical observations}

In this section, we suppose that all flows passing through a queue $m$ have the same maximum bit rate $r^{(m)}$. In contrast with the previous approach, we treat here the system as an one entity by evaluating firstly the average total number of flows passing through the whole system. Then, exploiting this result and using numerical results issued from some event simulations that capture the evolution of the average number of flows per queue in function of the weight assigned to it, we deduce an analytical explicit mathematical expression to evaluate the flow-level performance metrics for such system.

\subsection{1 | Evaluation of the average total number of flows}

Since each lost packet is sent again until it reaches the destination, looking at flow-level, it is assumed that the total traffic load is conserved in the system (there is no loss). For a best-effort system, the capacity of the link will be equally shared to carry the total traffic load. For a CBWFQ system, the same capacity will be shared in a differentiated manner to carry the same total traffic load. This leads to the fact that the mean total number of flows is approximately conserved between the two systems. It is equivalent to say that the mean total number of flows passing through our CBWFQ system is the same of an equivalent system that does not use CBWFQ policy 23 . We consider, for example, a link of capacity $C=50$ shared by three elastic flow classes such that $d_{1}=2, d_{2}=5$ and $d_{3}=10$. In figure 2 we compare the numerical result of the average total number of flows passing through a three CBWFQ system, such as $w_{1}=0.6, w_{2}=0.3$, and $w_{3}=0.1$, and the average total number of flows for a best-effort system, using the balanced fairness allocation (exploiting approximation 11), for different values of $\theta$. The numerical results are given by the resolution of a three-dimensional Markov chain modelling the arrival and departure of flows in the system. We assume that $\theta_{1}=\theta_{2}=\theta_{3}=\theta / 3$ and each queue is dedicated for one class. We can note that for values of $\theta / C$ inferior or equal to 0.8 , the error rate is practically negligible. This error rate increases in areas close to the instability zone, but we can always assume that the average total number of flows in the system is conserved. We can write then:

$$
\sum_{m=1}^{M} E_{m}^{C B W F Q} \approx \sum_{i \in E} E\left[x_{i}\right]
$$

Where $E_{m}^{C B W F Q}$ refers to the average total number of active flows passing through the queue number $m$ under the CBWFQ system and $E\left[x_{i}\right]$ is given by 11 . In the following, we denote by $E^{B E}$ the average total number of flows in best-effort system:

$$
E^{B E}=\sum_{i \in E} E\left[x_{i}\right]
$$

The average total number of flows in our CBWFQ system is then considered to be constant, and it is independent to the configuration of queue's weights. Therefore, when the weight of a queue $m$ tends to zero, it does not mean that this queue will be 


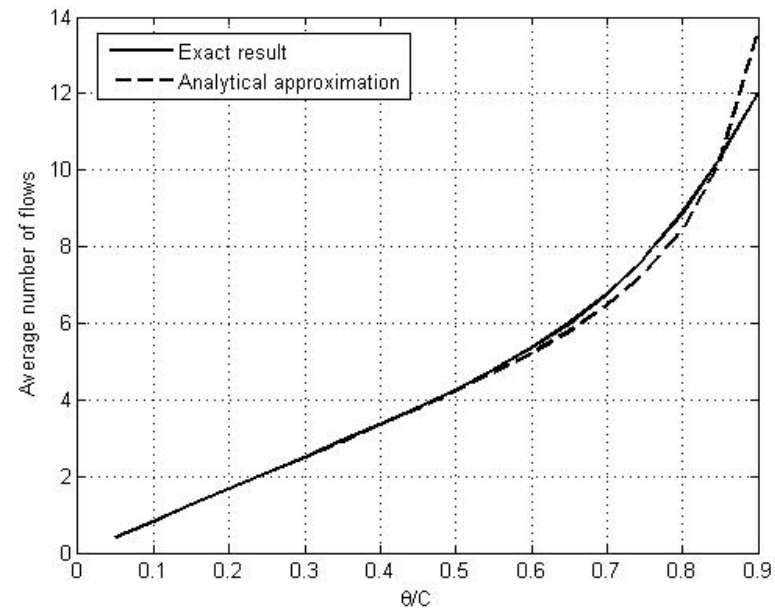

FIGURE 3 Comparison between the analytic and the exact result of the average number of flows for the first queue.

prevented from resources, but that it will be served after serving the other queues which are treated independently to the traffic of the queue $m$. These queues will then compose a second CBWFQ system of $M-1$ queues. Using 32 the average total number of flows for this system is given by:

$$
E^{B E / \theta-\theta_{m}} \approx \sum_{i \in E / E_{m}} E\left[x_{i}\right]
$$

Where $E\left[x_{i}\right]$ is given by 11

As a result, the average number of flows passing through the queue $m$ is obtained by:

$$
E_{m / w_{m} \rightarrow 0}^{C B W F Q} \approx E^{B E}-E^{B E / \theta-\theta_{m}}
$$

Considering the same previous example and assuming that $w_{1}=0, w_{2}=0.7$ and $w_{3}=0.3$, we compare in figure 3 the analytic result, given by 35, and the numerical result, given by the resolution of the three-dimensional Markov chain, in terms of the average number of flows of the first queue for different values of $\theta$. It is clear that the two results are very close and the error rate is practically negligible when the total load doesn't exceed $0.85 \%$ of the capacity.

\subsection{2 | Evaluating the average total number of flows for each queue: General case}

As we showed in ${ }^{23}$ for a system of two CBWFQ queues carrying traffic with identical maximum bit rate, the evolution of the average total number of flows for each queue $m$ in function of its weight can be estimated by the expression 36 where $\beta_{1}, \beta_{2}$ and $\beta_{3}$ are calculated according the system parameters and $\alpha$ is numerically adjusted to 3 . Our numerical observations show that this assumption remains true even for a greater number of queues or for different limit rates per queue.

$$
E_{m}^{C B W F Q} \approx \frac{\beta_{1}}{\beta_{2}+w_{m}^{\alpha}}+\beta_{3}
$$

The main goal of this section is to extend the results presented in ${ }^{23}$ for a system of two CBWFQ queues carrying an identical limit rate flows to a general case of $M$ queues and heterogeneous rate limits (we suppose that each queue is exploited by flows with the same maximum bit rate).

Using 36, the expression 35 can be written as follows:

$$
E_{m / w_{m} \rightarrow 0}^{C B W F Q} \approx \frac{\beta_{1}}{\beta_{2}}+\beta_{3}
$$

When the weight of a queue $m$, tends toward 1 , it can be considered as a priority queue ${ }^{44}$. Hence, the load $\theta_{m}$ is supposed to exploit all the capacity of the link. Let $E_{m / w_{m} \rightarrow 1}^{C B W F Q}$ the average number of flows passing through this queue in this case:

$$
E_{m / w_{m} \rightarrow 1}^{C B W F Q} \approx \frac{\beta_{1}}{\beta_{2}+1}+\beta_{3}
$$


$E_{m / w_{m} \rightarrow 1}^{C B W F}$ is deduced from 6 by replacing $\theta$ by $\theta_{m}$ and $r$ by $r^{(m)}$.

When the weight of each queue $m$ is equal to $1 / M$, the link capacity is supposed to be equally shared among the queues, and we are practically in the best-effort case. Therefore, we have:

$$
E_{m / w_{m}=1 / M}^{C B W F} \approx \sum_{i \in E_{m}} E\left[x_{i}\right]
$$

with $E\left[x_{i}\right]$ is given by 11 Using 36 we obtain:

$$
E_{m / w_{m}=1 / M}^{C B W F Q} \approx \frac{\beta_{1}}{\beta_{2}+(1 / M)^{\alpha}}+\beta_{3}
$$

The average total number of active flows in each queue can be then approximated by 36 where $\beta_{1}, \beta_{2}$ and $\beta_{3}$ are given from 37, 38 and 40 as follows:

$$
\begin{gathered}
\beta_{1} \approx\left(E_{m / w_{m}=1 / M}^{C B W F Q}-\beta_{3}\right)\left(\left(\frac{1}{M}\right)^{\alpha}+\beta_{2}\right) \\
\beta_{2} \approx \frac{\delta_{1}}{\left(M^{\alpha}-1\right) \delta_{2}-\delta_{1}} \\
\beta_{3} \approx E_{m / w_{m}=1 / M}^{C B W F Q}-\frac{1+\beta_{2}}{1-(1 / M)^{\alpha}} \delta_{1}
\end{gathered}
$$

With:

$$
\delta_{1}=E_{m / w_{m}=1 / M}^{C B W F Q}-E_{m / w_{m} \rightarrow 1}^{C B W F Q}
$$

And:

$$
\delta_{2}=E_{m / w_{m} \rightarrow 0}^{C B W F Q}-E_{m / w_{m}=1 / M}^{C B W F Q}
$$

Since all flows passing through a queue $m$ are supposed to have the same maximum bit rate, the average number of flows for each class $i \in E_{m}$ can be deduced from 10 as follows:

$$
E\left[x_{i}\right]=\frac{\rho_{i}}{\theta_{m}} E_{m}^{C B W F Q}
$$

\subsection{3 | Testing the accuracy of the second approach}

To examine the accuracy of the proposed analysis in which rely the second approach, we consider a scenario of capacity $C=50$ $(M$ bits $/$ Second $)$ shared by three TCP flow classes such that $d_{1}=5, d_{2}=4$ and $d_{3}=2($ all limit rates are in $(M$ bits $/$ Second $)$ ). Let $M=3$ and each queue is used by a single class of flows. In figure 4 we compare, in terms of the average number of flows passing through the first queue, our approximation 36 with the exact result given by the numerical resolution of the multidimensional Markov chain in function of the weight assigned to the first queue for different values of $\theta$. Our comparison is done for two different values of the ratio $\theta_{1} / \theta$, assuming that the other queues have the same contribution in the total load. For the numerical resolution, we assume that $w_{2}=3 w_{3}=3 / 4\left(1-w_{1}\right)$. The results presented in this figure show that the exact values of $E_{1}^{C B W F Q}$ are very close to those given by 36 . which confirms the good behaviour of our approximation. However, our approximation seems to be more accurate when the system is far for the instability regime. In fact, the error rate is practically negligible when the load is inferior or equal to $70 \%$ of the capacity. When the system is very loaded, the assumption of the preservation of the average total number of flows is not very accurate, and then the error rate increases a little bit.

\section{6 | SIMULATIONS AND VALIDITY OF THE ANALYTIC APPROACHES}

In this section, we aim to compare the proposed analytic approaches with the real behaviour of TCP traffic in order to verify its validity. We simulate then with NS 2 the case of a link of capacity $C=100$ ( $M$ bits $/$ Second $)$ shared by four TCP flow classes. We assume that at the entry of the link, there are four CBWFQ queues and each queue is exploited by only a single class of flows. Let $w_{1}=0.5, w_{2}=0.3, w_{3}=0.15$ and $w_{4}=0.05$.

Each TCP flow is used to transfer a series of packets of 1000 bytes representing a document of a certain size. The flow size distribution of each class is supposed to be exponential. The application we have chosen to be implemented for elastic traffic is FTP (File Transfer Protocol). We assumed that there is no additional time added by the link. 


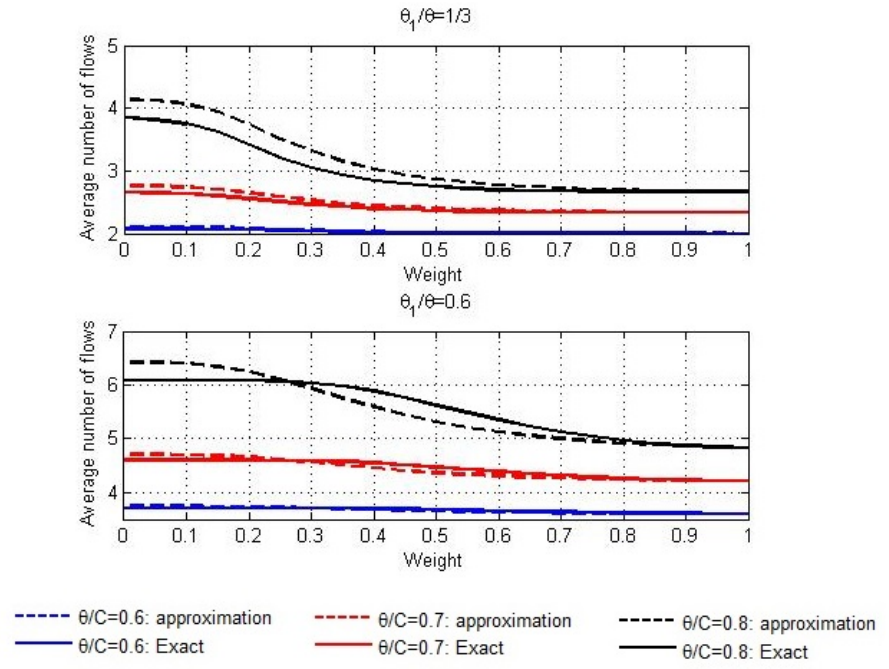

FIGURE 4 Comparison between the analytic and the exact result of the average number of flows for the first class in function of the weight assigned to it

Each simulation point is the average of the results of ten simulations with variable values of seed. Each simulation last two hours, in which we simulate the arrival and the departure of thousands of flows.

In the figures $5,6 \quad 7$ and 8 , the two analytic approaches are compared with the simulation results in terms of the average queue throughput (each queue is used by a single class of flows) respectively for four different scenarios. In the first scenario, we simulate the case where the classes of flows have different limit rates, such as $d_{1}=1, d_{2}=2, d_{3}=3$ and $d_{4}=5$ in (Mbits/Second), and different contributions in the total load, such as the ratio $\theta_{m} / w_{m} C, m=1 . .4$, is always the same. The second scenario is a particular case for the first scenario supposing that all classes of flows have an identical limit rate $r=5$ (Mbits /Second). In the third and the fourth scenarios, we repeat the same previous scenarios by assuming that all classes of flows have the same contribution in total load.The accuracy of our proposed results is measured by the error rate defined as:

$$
\text { ErrorRate }(\%)=100 \frac{\mid \text { Analytic Result }- \text { SimulationResult } \mid}{\text { SimulationResult }}
$$

At first, it is necessary to note that, for all simulated cases, the two analytic results are much more accurate for non very loaded system, with an error rate globally inferior to 5\% when the total offered load does not exceed $70 \%$ of the capacity, which proves the efficiency of our two analytic approaches. It is also important to note that our analytical and numerical results match with ${ }^{43}$ where the authors concluded that CBWFQ has practically no effect in an under-loaded system. Our simulations prove that, independently of the queue's weight, flows tend to have its maximum bit rate when the system is not very loaded. As it is showed in the figures, the service differentiation through CBWFQ policy is more significant when the load becomes critical (more than $70 \%$ of the capacity).

For the first and second scenarios, and for non-loaded zones, the two approaches give two close results for the first two queues with an error rate inferior to $3 \%$. This is explained by the fact that with a high or medium weight, the queue is not very coupled with the other queues making the estimation of its decoupled service rate more accurate. However, for the other queues, the second approach is more accurate than the first one, which overestimates the average queue throughput. Indeed, for the second scenario, the error rate at the third queue seems to be negligible (less than 0.4\%) with the second approach, while it exceeds $4 \%$ with the first approach and it is less than $4.5 \%$ for the fourth queue with the second approach while it reached $8 \%$ with the first one. This is explained by the fact that for very low weights, the second approach is based on the explicit mathematical expressions 32 and 35, which has already proved their accuracy especially for non-loaded system.

In the last two scenarios, the second approach is more relevant to estimate the performance of elastic traffic in such system. In fact, with this approach, the error rate between the analytic and simulated results is negligible when the total load is less than $75 \%$ of the link capacity and it does not exceed $5 \%$ when the system tends to the instability regime. For the last queue with a 
very low weight, the first approach can't capture the real coupling between the queues and then it is unable to give an accurate and reasonable results: According to this approach, the fourth queue did not receive sufficient additional resources to circulate the important traffic passing through it. Thus, for some states, the average load of this queue remains higher than its average capacity, and then, the average throughput for all flows passing through it is supposed to rapidly tends to zero.

In general, and for all simulated cases, the second approach provides very good results for critical values of load. This is particularly interesting given that the CBWFQ mechanism is more efficient for very loaded systems.
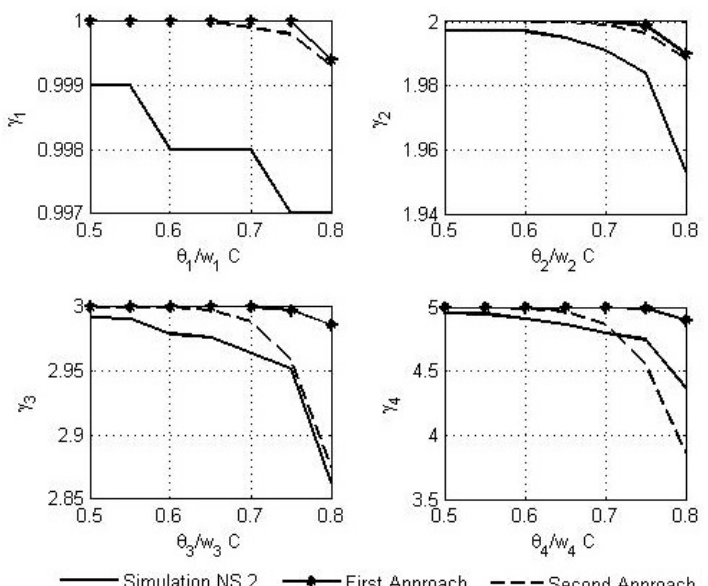

First Approach - - Second Approach

FIGURE 5 Comparison in terms of the average throughput per queue between the two analytic approaches and the simulations results: First scenario
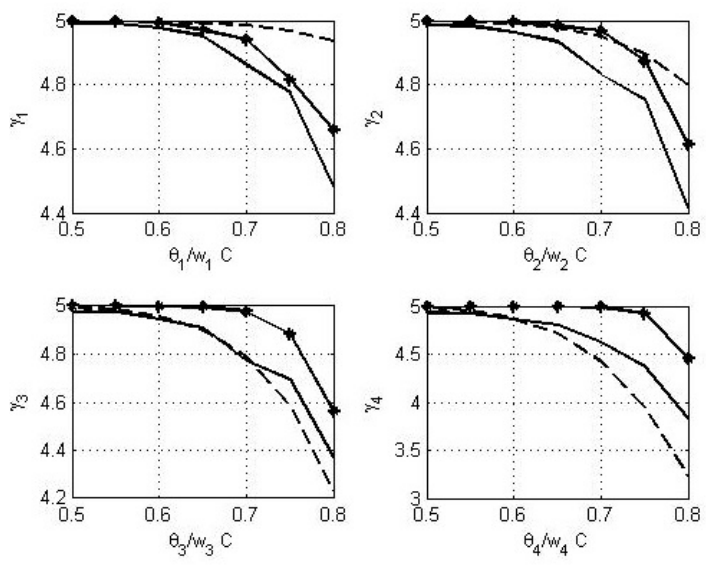

$\longrightarrow$ Simulation NS $2 \longrightarrow$ First Approach $\quad---$ Second Approach

FIGURE 6 Comparison in terms of the average throughput per queue between the two analytic approaches and the simulations results: Second scenario

\section{7 | CONCLUSION}

In this paper we propose two new fluid approaches to evaluate the performance of elastic traffic under a CBWFQ system. The key characteristic of our analysis is to rely on some approximations for balanced fairness allocation, which is often considered 

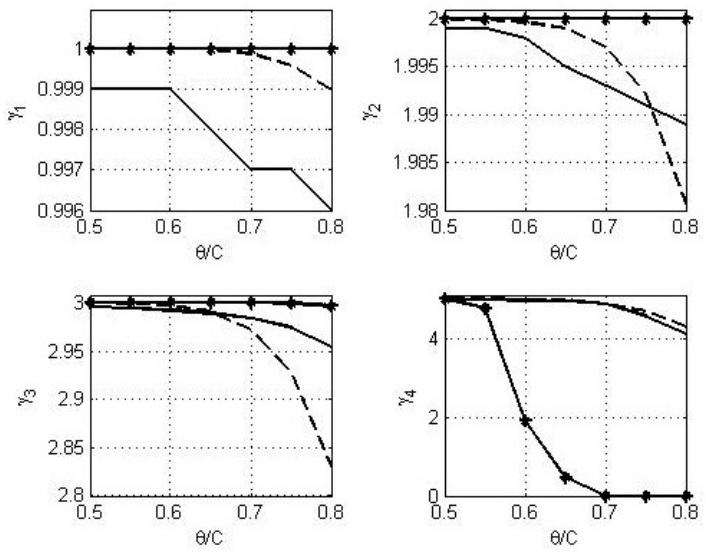

- Simulation NS2

$\longrightarrow$ First Approach

--- Second Approach

FIGURE 7 Comparison in terms of the average throughput per queue between the two analytic approaches and the simulations results: Queues with same load and different limit rates
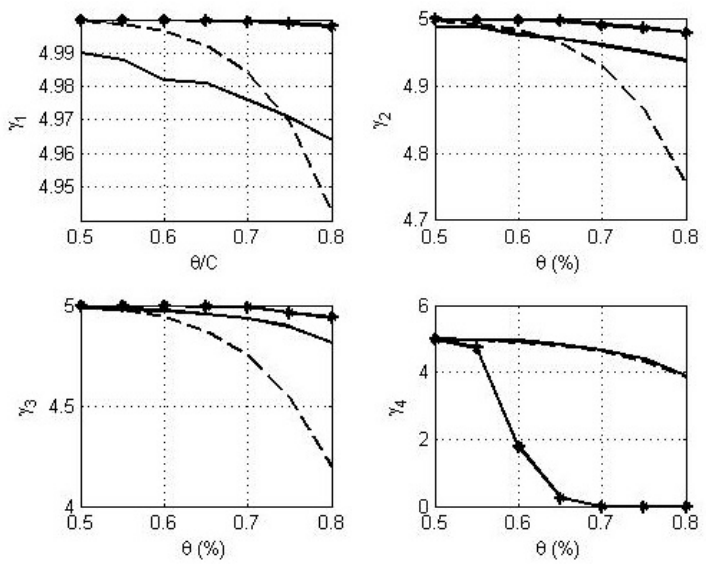

$\longrightarrow$ Simulation NS $2 \longrightarrow$ First Approach $\quad---$ Second Approach

FIGURE 8 Comparison in terms of the average throughput per queue between the two analytic approaches and the simulations results: Queues with same load and identical limit rates

as an efficient tool to estimate the bandwidth sharing among elastic traffic under best-effort system, to highlight the coupling aspect between queues in such system.

The first approach aims to decouple the system into different independent virtual links, each with an average mean capacity while the second is mainly based on some numerical observations for the evolution of the average total number of flows for a queue in function of the weight assigned to it. Detailed packet level simulations are used to test the efficiency and the accuracy of our approaches.

Although the first approach may be relevant to estimate the mean capacity received by each virtual link (or the mean service rate received by each queue), our simulations show that this approach can't give accurate results for queues with low weight, where the coupling with other queues seems to be strong. In the second approach, which globally provides more accurate results than the first one, the average number of flows for each class passing through a queue is deduced from the average total flows for this queue. For the moment, this can be only estimated when all elastic traffic carried by this queue has the same limit rate by using the relation 10 Further works are needed to increase the accuracy of this approach and to generalise it to the case where elastic flow classes with different limit rates can pass through the same queue. 


\section{APPENDIX}

\section{A PROOF OF THE EQUATION (17)}

In this section we deal with independent virtual links, each with capacity $w_{k} C, \forall 1 \leq k \leq M$. We aim to calculate $\alpha_{m}^{(1)}$, the average capacity received by the queue $m$ from the other queues when all other queues doesn't use its minimum capacity. Let $n^{(k)}=x^{(k)} d^{(k)}, \forall 1 \leq k \leq M$.

We denote by $\overline{\sum_{\substack{0 \leq n^{(k)}<w_{k} C \\ 1 \leq k \leq M}}}$ the multiple summation of length $M-1$ on $n^{(k)}, 1 \leq k \leq M, k \neq m$. For example, if $1 \leq m<M-1$, this notation refers to:

$$
\overline{\sum_{\substack{0 \leq n^{k}<w_{k} C \\ 1 \leq k \leq M \\ k \neq m}}}=\sum_{\substack{0 \leq n^{(1)}<w_{1} C \\ C}} \sum_{0 \leq n^{(2)}<w_{2} C} . . \sum_{\substack{0 \leq n^{(m-1)}<w_{m-1} C \\ 0 \leq n^{(m+1)}<w_{m+1} C}} . . \sum_{0 \leq n^{(M)}<w_{M} C}
$$

Then, we have these two notations:

$$
\begin{aligned}
& \sum_{\substack{0 \leq n^{(k)}<w_{k} C \\
1 \leq k \leq M \\
k \neq m}} \sum_{f} n^{(f)}=\sum_{\substack{0 \leq n^{(1)}<w_{1} C \\
C}} \sum_{0 \leq n^{(2)}<w_{2} C} . . \sum_{\substack{0 \leq n^{(m-1)}<w_{m-1} C \\
C}} \sum_{0 \leq n^{(m+1)}<w_{m+1} C}{ }^{.} \sum_{0 \leq n^{(M)}<w_{M} C}\left(n^{(1)}+n^{(2)}+\ldots .+n^{(m-1)}+n^{(m+1)}+\ldots+n^{(M)}\right) \\
& \sum_{\substack{0 \leq n^{(k)}<w_{k} C \\
1 \leq k \leq M \\
k \neq m}} \prod_{h} \pi\left(n^{(h)}\right)=\sum_{0 \leq n^{(1)}<w_{1} C} \sum_{0 \leq n^{(2)}<w_{2} C} . . \sum_{\substack{0 \leq n^{(m-1)}<w_{m-1} C\\
}} \sum_{0 \leq n^{(m+1)}<w_{m+1} C} . . \sum_{0 \leq n^{(M)}<w_{M} C}\left(\pi\left(n^{(1)}\right) \pi\left(n^{(2)}\right) \ldots \pi\left(n^{(m-1)}\right) \pi\left(n^{(m+1)}\right) \ldots \pi\left(n^{(M)}\right)\right)
\end{aligned}
$$

Using these notations, we have:

$$
\begin{aligned}
& \alpha_{m}^{(1)}=\sum_{\substack{0 \leq n^{(k)}<w_{k} C \\
1 \leq k \leq M \\
k \neq m}}\left[\left[\left(1-w_{m}\right) C-\sum_{j} n^{(j)}\right] \prod_{h} \pi\left(n^{(h)}\right)\right] \\
& =\sum_{\substack{(k) \notin B(k) \\
1 \leq k \leq M \\
k \neq m}}\left[\left[\left(1-w_{m}\right) C-\sum_{j} \sum_{i \in E_{j}} d_{i} x_{i}\right] \prod_{h} \pi\left(x^{(h)}\right)\right]
\end{aligned}
$$

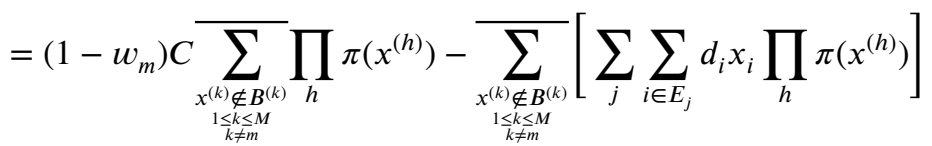

$$
\begin{aligned}
& =\left(1-w_{m}\right) C \prod_{\substack{1 \leq k \leq M \\
k \neq m}}\left[1-\pi\left(B^{(k)}\right)\right]-{\overline{\substack{x^{(k)} \notin B^{(k)} \\
1 \leq k \leq M \\
k \neq m}}}_{[}\left[\sum_{j} \sum_{i \in E_{j}} d_{i} x_{i} \prod_{h} \pi\left(x^{(h)}\right)\right] \\
& =\left(1-w_{m}\right) C \prod_{\substack{1 \leq k \leq M \\
k \neq m}}\left[1-\pi\left(B^{(k)}\right)\right]-{\overline{\substack{x^{(k) \notin B} \\
1 \leq k \leq M \\
k \neq m}}}_{\substack{(k) \\
k}}\left[\sum_{j} d_{i} x_{i} \pi\left(x^{(j)}\right) \prod_{h \neq j} \pi\left(x^{(h)}\right)\right] \\
& =\left(1-w_{m}\right) C \prod_{\substack{1 \leq k \leq M \\
k \neq m}}\left[1-\pi\left(B^{(k)}\right)\right]-\sum_{\substack{1 \leq j \leq M \\
j \neq m}} \sum_{x^{(j)} \notin B^{(j)}} \sum_{i \in E_{j}} d_{i} x_{i} \pi\left(x^{(j)}\right) \prod_{\substack{1 \leq h \leq M \\
h \neq m \\
h \neq j}}\left[1-\pi\left(B^{(h)}\right)\right]
\end{aligned}
$$

By adopting balanced fairness allocation on the virtual link $j$, we have for all $i \in E_{j}$, the following two relations $\frac{1645}{\text { : }}$. 


$$
\begin{gathered}
\phi_{i}\left(x^{(j)}\right)=\frac{\Phi\left(x^{(j)}-e_{i}^{(j)}\right)}{\Phi\left(x^{(j)}\right)} \\
\pi\left(x^{(j)}\right)=\pi(0)^{(j)} \Phi\left(x^{(j)}\right) \rho^{(j)^{\left(x^{(j)}\right)}}
\end{gathered}
$$

Where $\phi_{i}\left(x^{(j)}\right)$ is the total capacity allocated to class-i flows in the state $x^{(j)}, \Phi\left(x^{(j)}\right)$ is the balance function corresponding to the balanced fairness allocations and $e_{i}^{(j)}$ is a vector of size $\operatorname{Card}\left\{E_{j}\right\}$ with 1 in component $\mathrm{i}$ and 0 elsewhere.

Thus we have:

$$
\begin{aligned}
\pi\left(x^{(j)}-e_{i}^{(j)}\right) & =\pi(0)^{(j)} \Phi\left(x^{(j)}-e_{i}^{(j)}\right) \rho^{(j)}\left(^{\left(x^{(j)}-e_{i}^{(j)}\right)}\right. \\
& =\frac{\phi_{i}\left(x^{(j)}\right)}{\rho_{i}} \pi(0)^{(j)} \Phi\left(x^{(j)}\right) \rho^{(j) x^{(j)}} \\
& =\frac{\phi_{i}\left(x^{(j)}\right)}{\rho_{i}} \pi\left(x^{(j)}\right)
\end{aligned}
$$

So:

$$
\pi\left(x^{(j)}\right)=\frac{\rho_{i}}{\phi_{i}\left(x^{(j)}\right)} \pi\left(x^{(j)}-e_{i}^{(j)}\right)
$$

For $0 \leq n^{(j)}<w_{j} C$ we have:

$$
\pi\left(x^{(j)}\right)=\frac{\rho_{i}}{x_{i} d_{i}} \pi\left(x^{(j)}-e_{i}^{(j)}\right)
$$

For all $i \in E_{j}$, We have then:

$$
\begin{aligned}
\sum_{x^{(j)} \notin B^{(j)}} x_{i} \pi\left(x^{(j)}\right) & =\frac{\rho_{i}}{d_{i}} \sum_{x^{(j)} \notin B^{(j)}} \pi\left(x^{(j)}-e_{i}^{(j)}\right) \\
& =\frac{\rho_{i}}{d_{i}} \sum_{0 \leq n^{(j)}<w_{j} C-d_{i}} \pi\left(x^{(j)}\right) \\
& =\frac{\rho_{i}}{d_{i}}\left(1-\pi\left(B_{i}^{(j)}\right)\right)
\end{aligned}
$$

From A6 and A11 we conclude:

$$
\alpha_{m}^{(1)}=\left(1-w_{m}\right) C \prod_{\substack{1 \leq k \leq M \\ k \neq m}}\left[1-\pi\left(B^{(k)}\right)\right]-\sum_{\substack{1 \leq j \leq M \\ j \neq m}} \sum_{i \in E_{j}} \rho_{i}\left[1-\pi\left(B_{i}^{(j)}\right)\right] \prod_{\substack{1 \leq h \leq M \\ h \neq m \\ h \neq j}}\left[1-\pi\left(B^{(h)}\right)\right]
$$

\section{B PROOF OF THE EQUATION (18)}

In this section we deal with independent virtual links, each with capacity $w_{k} C, \forall 1 \leq k \leq M$. We aim to calculate $\alpha_{m}^{(2)}$, the average capacity received by the queue $m$ from the other queues when there is at least one other queue that will share the total remaining capacity with this queue. Let $t$ the number of the queues that will share with the queue $m$ the remaining capacity in the other queues. Since it is supposed here that there is always at least one queue that doesn't use the totality of its minimum capacity, $t$ is always between 1 and $M-2$. Let $n^{(k)}=x^{(k)} d^{(k)}, \forall 1 \leq k \leq M$. 
Using the same notations as the precedent proof, we have:

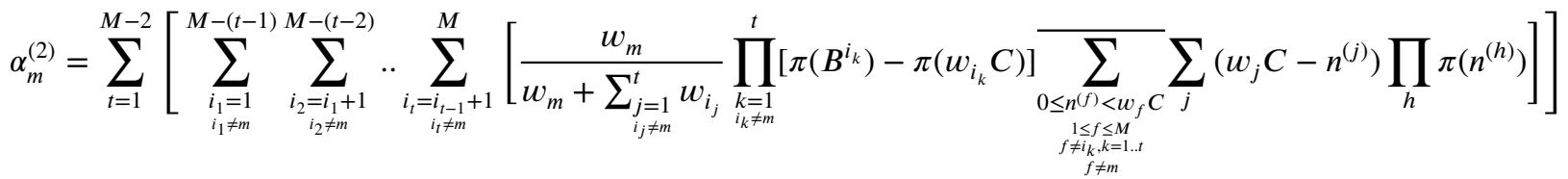

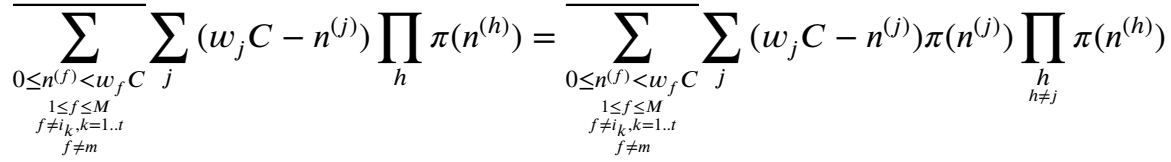

$$
\begin{aligned}
& =\sum_{\substack{1 \leq f \leq M \\
f \neq i, k=1 . t \\
f \neq m}} C_{f}^{(R)} \prod_{\substack{1 \leq h \leq M \\
h \neq m \\
h \neq f \\
h \neq i_{k}, k=1 . . t}}\left[1-\pi\left(B^{(h)}\right)\right]
\end{aligned}
$$

We deduce then:

$$
\alpha_{m}^{(2)}=\sum_{t=1}^{M-2}\left[\sum_{\substack{i_{1}=1 \\ i_{1} \neq m}}^{M-(t-1)} \sum_{\substack{i_{2}=i_{1}+1 \\ i_{2} \neq m}}^{M-(t-2)} \cdot \sum_{\substack{i_{t}=i_{t-1}+1 \\ i_{t} \neq m}}^{M}\left[\frac{w_{m}}{w_{m}+\sum_{\substack{j=1 \\ i_{j} \neq m}}^{t} w_{i_{j}}} \prod_{\substack{k=1 \\ i_{k} \neq m}}^{t}\left[\pi\left(\boldsymbol{B}^{i_{k}}\right)-\pi\left(w_{i_{k}} C\right)\right] \sum_{\substack{1 \leq f \leq M \\ f \neq i_{k}, k=1 . t \\ f \neq m}} C_{f}^{(R)} \prod_{\substack{1 \leq h \leq M \\ \text { and } \\ h \neq m \\ h \neq i_{k}, k=1 . t}}\left[1-\pi\left(\boldsymbol{B}^{(h)}\right)\right]\right]\right]
$$

\section{PROOF OF THE EQUATION (23)}

In this section we deal with independent virtual links, each with capacity $w_{f} C, \forall 1 \leq f \leq M$. Let $n^{(f)}=x^{(f)} d^{(f)}$.

The average remaining capacity in each virtual link $f$ is given by:

$$
\begin{aligned}
C_{f}^{(R)} & =\sum_{0 \leq n^{(f)}<w_{f} C}\left(w_{f} C-n^{(f)}\right) \pi\left(n^{(f)}\right) \\
& =\sum_{0 \leq n^{(f)}<w_{f} C} w_{f} C \pi\left(n^{(f)}\right)-\sum_{0 \leq n^{(f)}<w_{f} C} n^{(f)} \pi\left(n^{(f)}\right) \\
& =\sum_{x^{(f)} \notin B^{(f)}} w_{f} C \pi\left(x^{(f)}\right)-\sum_{i \in E^{(f)}} d_{i} \sum_{x^{(f)} \notin B^{(f)}} x_{i} \pi\left(x^{(f)}\right) \\
& =w_{f} C\left(1-\pi\left(B^{(f)}\right)\right)-\sum_{i \in E^{(f)}} d_{i} \sum_{x^{(f)} \notin B^{(f)}} x_{i} \pi\left(x^{(f)}\right)
\end{aligned}
$$

By adopting balanced fairness allocation on the virtual link $f$ and using A11 we deduce:

$$
C_{f}^{(R)}=w_{f} C\left(1-\pi\left(B^{(f)}\right)\right)-\sum_{i \in E^{(f)}} \rho_{i}\left(1-\pi\left(B_{i}^{(f)}\right)\right.
$$

\section{PROOF OF THE EQUATION (25)}

In this section we deal with an independent virtual link $f$ with capacity $w_{f} C$. Let $\pi\left(\left|x_{f}\right|\right)$ the probability that we have $\left|x_{f}\right|$ connections in the queue $f$ and $r^{(f)}$ the maximum bit rate of the flows passing through this queue. The average remaining capacity of the virtual link number $f$ is given by:

$$
\begin{aligned}
& C_{f}^{(R)}=\sum_{\left|x^{(f)}\right| \leq N^{(f)}}\left(w_{f} C-\left|x^{(f)}\right| r^{(f)}\right) \pi\left(x^{(f)}\right) \\
& \sum_{\left|x^{(f)}\right| \leq N^{(f)}} w_{f} C \pi\left(x^{(f)}\right)=w_{f} C\left(1-\pi\left(B^{(f)}\right)\right. \\
& \left.+\pi\left(\left|x_{f)}\right|=N^{(f)}\right)\right)
\end{aligned}
$$


As we showed in APPENDIX A, for $\left|x^{(f)}\right| \leq N^{(f)}$ we have :

$$
\pi\left(x^{(f)}\right)=\frac{\rho_{i}}{x_{i} r^{(f)}} \pi\left(x^{(f)}-e_{i}^{(f)}\right)
$$

Therefore:

$$
\begin{aligned}
\sum_{\left|x^{(f)}\right| \leq N^{(f)}}\left|x^{(f)}\right| r^{(f)} \pi\left(x^{(f)}\right) & =r^{(f)} \sum_{\left|x^{(f)}\right| \leq N^{(f)}} \sum_{i \in E_{f}} x_{i} \pi\left(x^{(f)}\right) \\
& =\sum_{\left|x^{(f)}\right| \leq N^{(f)}} \sum_{i \in E_{f}} \rho_{i} \pi\left(x^{(f)}-e_{i}^{(f)}\right) \\
& =\theta_{f} \sum_{\left|x^{(f)}\right| \leq N^{(f)}-1} \pi\left(x^{(f)}\right) \\
& =\theta_{f}\left(1-\pi\left(B^{(f)}\right)\right)
\end{aligned}
$$

And then, we conclude:

$$
C_{f}^{(R)}=\left(w_{f} C-\theta_{f}\right)\left(1-\pi\left(B^{(f)}\right)\right)+w_{f} C \pi\left(N^{(f)}\right)
$$

\section{References}

1. Jursonovics T, Imre S. Quality-based charging solutions for wireless multimedia services. International Journal of Network Management. 2014;24(5):357-401.

2. Foteinos V, Tsagkaris K, Peloso P, Ciavaglia L, Demestichas P. Energy-aware traffic allocation to optical lightpaths in multilayer core networks. International Journal of Network Management. 2015;25(6):394-417.

3. Shim KS, Ham JH, BD Sija, Kim M. S.. Application traffic classification using payload size sequence signature. International Journal of Network Management. 2017;27(5).

4. Bian Y, Liu B, Li Y, Gao J. Characterizing network traffic behaviour using granule âĂ $\check{R}$ based association rule mining. International Journal of Network Management. 2016;26(4):308-329.

5. Networking Index, Cisco Visual . Forecast and methodology, 2016-2021, white paper. San Jose, CA, USA. 2016;1.

6. An explosion of online video could triple bandwidth consumption again in the next five years https://www.recode.net/2017/ 6/8/15757594/future-internet-traffic-watch-live-video-facebook-google-netflix Accessed: 2012-12-13; .

7. Brosh E, Baset S A, Misra V, Rubenstein D, Schulzrinne H. The delay-friendliness of TCP for realtime traffic. IEEE/ACM Transactions On Networking. 2010;18(5):1478-1491.

8. Wang Bing, Kurose Jim, Shenoy Prashant, Towsley Don. Multimedia streaming via TCP: An analytic performance study. ACM Transactions on Multimedia Computing, Communications, and Applications (TOMM). 2008;4(2):16.

9. Biernacki Arkadiusz, Tutschku Kurt. Performance of HTTP video streaming under different network conditions. Multimedia Tools and Applications. 2014;72(2):1143-1166.

10. Aweya J, Ouellette M, Montuno D Y. Weighted proportional window control of TCP traffic. International Journal of Network Management. 2001;11(4):213-242.

11. Wang Z, Basu A. Resource allocation for elastic traffic: Architecture and mechanisms. In: Network Operations and Management Symposium, 2000. NOMS 2000. 2000 IEEE/IFIP; April 10-14, 2000; Honolulu, Hawaii, USA.

12. Dan A, Ludwig H, Pacifici G. Web service differentiation with service level agreements. White Paper, IBM Corporation. $2003 ; 3$. 
13. Balogh T, Medvecky M. Performance evaluation of WFQ, WF2Q+ and WRR queue scheduling algorithms. In: Proceeding of the 34th International Conference on Telecommunications and Signal Processing:136-140; August 18-20, 2011; Budapest, Hungary.

14. Al-Sawaai A, Awan I, Fretwell R. Performance Evaluation of Weighted Fair Queuing System Using Matrix Geometric Method. NETWORKING 2009. 2009;:66-78.

15. Shortle John F, Fischer Martin J. Approximation for a two-class weighted fair queueing discipline. Performance Evaluation. 2010;67(10):946-958.

16. Bonald T, Massoulié L, Proutière A, Virtamo J. A queueing analysis of max-min fairness, proportional fairness and balanced fairness. Queueing systems. 2006;53(1):65-84.

17. Fischer M J, Masi D M B, Shortle J F. Simulating the performance of a class-based weighted fair queueing system. In: Proceedings of the 40th Conference on Winter Simulation:2901-2908; December 07-10, 2008; Miami, FL, USA.

18. Bevilacqua Masi D M, Fischer M J, Garbin D A. Modeling the performance of low latency queueing for emergency telecommunications. In: Proceedings of the 39th conference on Winter simulation: 40 years! The best is yet to come:2266-2275; December 09 - 12, 2007; Washington, DC, USA.

19. Horváth G, Telek M. An approximate analysis of two class WFQ systems. In: Proceeding of the Workshop on Preformability Modeling of Computer and Communication Systems-PMCCS:43-46; September 2003; Arlington, IL, USA.

20. Dekeris B, Adomkus T, Budnikas A. Analysis of QoS assurance using weighted fair queueing (WQF) scheduling discipline with low latency queue (LLQ). In: Proceedings of the 28th International Conference on Information Technology Interfaces:507-512; June 19-22, 2006; Cavtat, Croatia.

21. Ashour M, Le-Ngoc T. Performance analysis of weighted fair queues with variable service rates. In: Proceedings of the International Conference on Digital Telecommunications ICDT 2006:51-51; August 29-31, 2006; Cap Esterel,Cote D'azur, France.

22. Presti F L, Zhang Z L, Towsley D. Bounds, approximations and applications for a two-queue GPS system. In: Proceeding of the INFOCOM'96. Fifteenth Annual Joint Conference of the IEEE Computer Societies. Networking the Next Generation. Proceedings IEEE, vol. 3: :1310-1317; March 24-28,1996; San Francisco, CA, USA.

23. Boussada M E H, Garcia J M, Frikha M. Numerical Key Results for Studying the Performance of TCP Traffic under Class-Based Weighted Fair Queuing System. Journal of Communication and Computer. 2016;13:195-201.

24. Barakat C, Thiran P, Iannaccone G, Diot C, Owezarski P. Modeling Internet backbone traffic at the flow level. IEEE Transactions on Signal processing. 2003;51(8):2111-2124.

25. Hassan H. Modélisation et analyse de performances du trafic multimédia dans les réseaux hétérogènes. PhD thesisUniversité Paul Sabatier-Toulouse III2006.

26. Olivier B, Al Sheikh A, Garcia J M. Flow-Level Modelling of TCP Traffic Using GPS Queueing Networks. In: Proceeding of The 21st International Teletraffic Congress:1-8; September 15-17, 2009; Paris, France.

27. Bonald T, Haddad J P, Mazumdar R R. Congestion in large balanced multirate links. In: Proceedings of the 23rd International Teletraffic Congress:182-189; September 6-8, 2011; San Francisco, USA.

28. Bonald T, Comte C. The multi-source model for dimensioning data networks. Computer Networks. 2016;109:225-233.

29. Niculae A M. Mécanismes d'optimisation multi-niveaux pour IP sur satellites de nouvelle génération. PhD thesisUniversité de Toulouse2009.

30. Garcia J M, Boussada M E H. End-to-End Performance Evaluation of TCP Traffic under Multi-Queuing Networks. International Journal of Communications, Network and System Sciences. 2016;9(6):219-233. 
31. Massoulié Laurent, Roberts James W. Bandwidth sharing and admission control for elastic traffic. Telecommunication systems. 2000;15(1-2):185-201.

32. Bonald T, Virtamo J. Calculating the flow level performance of balanced fairness in tree networks. Performance Evaluation. 2004;58(1):1-14.

33. Bonald T, Proutiere A. Insensitive bandwidth sharing in data networks. Queueing systems. 2003;44(1):69-100.

34. Massoulié L. Structural properties of proportional fairness: stability and insensitivity. The Annals of Applied Probability. 2007;:809-839.

35. Cheikh H B. Evaluation et optimisation de la performance des flots dans les réseaux stochastiques à partage de bande passante. PhD thesisINSA de Toulouse2015.

36. Garcia J M, Boussada M E H. Evaluation des performances bout en bout du trafic TCP sous le régime «Équité Équilibrée». In: The 11th Performance Evaluation Workshop; March 15-17, 2016; Toulouse,France.

37. Bonald T, Penttinen A, Virtamo J. On light and heavy traffic approximations of balanced fairness. In: ACM SIGMETRICS Performance Evaluation Review, vol. 34: :109-120; 2006.

38. Bonald T, Virtamo J. A recursive formula for multirate systems with elastic traffic. IEEE Communications Letters. 2005;9(8):753-755.

39. Ben Cheikh Henda. Integration of streaming and elastic traffic: Modeling and Performance Analysis. In: The 9th EAI International Conference on Performance Evaluation Methodologies and Tools:65-68ICST (Institute for Computer Sciences, Social-Informatics and Telecommunications Engineering); December 14 - 16, 2015; Berlin, Germany.

40. Borst S, Mandjes M, Uitert M. Generalized processor sharing with light-tailed and heavy-tailed input. IEEE/ACM Transactions on Networking (TON). 2003;11(5):821-834.

41. Lapiotis G, Panwar S. Quality of service analysis of shared buffer management policies combined with generalized processor sharing. In: Proceeding of the Global Telecommunications Conference, 1999. GLOBECOM'99, vol. 1: :37-43; December 5-9, 1999; Rio de Janeiro, Brazil.

42. Ashour M, Le-Ngoc T. Performance of weighted fair queuing systems with long range dependent traffic inputs. In: Canadian Conference on Electrical and Computer Engineering, 2005:1002-1005; May 1-4, 2005; Saskatoon, SK, Canada.

43. Bonald T, Roberts J W. Congestion at flow level and the impact of user behaviour. Computer networks. 2003;42(4):521-536.

44. Bockstal C, Garcia J M, Brun O. Approximation du régime stationnaire d'un système wfq. In: ; May 26-28,2004; Batz-surMer, France.

45. Bonald T, Proutiere A, Roberts J, Virtamo J. Computational aspects of balanced fairness. Teletraffic Science and Engineering. 2003;5:801-810. 